\title{
Bridging primary and secondary care for people with learning disabilities
}

\author{
Neill J. Simpson
}

\section{Definitions and terminology}

Definitions for learning disability vary depending on the context in which they are used (Box 1), as does the terminology (Box 2). In the UK, the term 'learning disability' was adopted in 1991 by the Department of Health. The term 'mental retardation' is used in the ICD-10 (World Health Organization, 1992). Fryers (1991) has commented on the need for different definitions in order to distinguish the context in which the term is used.

There are other developmental disabilities due to impairments of cognitive function, such as autism and related disorders. Some mental disorders of childhood, especially hyperkinetic syndrome, also cause developmental cognitive impairment. It is important for all doctors to be able to detect these conditions (Box 3 ), as some conditions are remediable or have associations with other health problems, and also to ensure access to services.

Cognitive impairment is often associated with social disadvantage and socially-disapproved behaviour, and words to describe it become pejorative. Unfamiliar, technical words become insults more rapidly than ordinary English, and it is unhelpful to use euphemisms. For this reason, the term 'learning disability' is probably best used by doctors when discussing disability with patients and their families. For other purposes, such as epidemiology, terms with more precise definition will still be required.
Box 1. What learning disability means in practice

'Arrested or incomplete development of mind' means that the impairment of mental development is established before adulthood. No age limit is given, except in DSM-IV (APA, 1994) which gives the 18th birthday as the latest date for the impairment to be established. Clinically, the age of leaving school is sometimes used.

'Significant impairment of intelligence' means that the person has difficulty in understanding new or complex information and learning new skills. Evidence may be drawn from psychometric assessment of performance on cognitive tasks such as the Wechsler Adult Intelligence Scale; or from reports by suitable informants such as teachers or parents.

'Significant impairment of social functioning' means that the person has not acquired all the abilities required to function independently in society. Key skills ('adaptive behaviours') are self-care, homemaking, communication, responding to social expectations, and responding to new demands ('coping'). Evidence may be drawn from self-report, observation, or informants' account. Assessment schedules are available, such as the Adaptive Behaviour Scale and the Vineland Social Maturity Scale. 
Box 2. Terminology

Mental retardation (ICD-10; WHO, 1992): defined as "a state of arrested or incomplete development of mind including significant impairment of intelligence and social functioning".

Learning disability (UK Department of Health): definition is the same as above.

Mental impairment (1) (Mental Health Act, 1983). Limited to people with learning disabilities who also have "abnormally aggressive or seriously irresponsible conduct".

Mental impairment (2) (Council Tax Regulations): requires impairment of intelligence and social functioning to be "permanent", and not necessarily due to "arrested or incomplete development of mind".

Special educational need (Education Act, 1981): a formal statement of special needs of pupils with learning disability and for other reasons. specialist advice, so that children with disabilities can obtain education and other services which could help them and their families. A good case can be made for systematic developmental screening in primary care, possibly using a checklist of indications for referral (Hall, 1984).

\section{Mild learning disability}

People with mild learning disability may find it hard to manage complex tasks, such as the household budget, or to adapt readily to new situations, such as bereavement, onset of illness, or other life events. If they have a supportive family and a stable lifestyle, and do not have complex disabilities, then they will probably manage without extra support from specialised services. Mild learning disability accounts for around $2 \%$ of the population, and around $80 \%$ of all people with learning disabilities. The majority of health problems will be dealt with in primary care, and GPs may not realise that some of their patients have mild learning disabilities.

\section{Severe learning disability}

People with severe learning disability usually need supervision or assistance with some of the skills required for independence. Most will require supervision by a responsible person on a daily basis, and they are likely to be detected as having special educational needs at school.

Box 3. Some developmental disabilities which should be detected in primary care

Hyperkinetic syndrome: may respond to medication. Associated with various neuro-developmental problems. Some cases may be caused by phenobarbitone.

Tourette's syndrome: may respond to medication. Associated with obsessivecompulsive features.

Autism (and related disorders): access to suitable education depends on early detection. Associated with epilepsy, depression, and behavioural problems.

Specific developmental disorders of speech and language, and other specific learning difficulties: access to suitable education depends on early detection. May indicate brain localisation. 


\section{Family needs}

Much of the information and advice that families require is straightforward and should be available in primary care, such as information about welfare rights and how to contact services and voluntary organisations. General health care should be provided, and many aspects of health promotion may be done by the primary health care team working with families. Sometimes specialist knowledge is essential, as for genetic counselling, where it is important that specialists inform GPs what the family has been told.

Families may need to be able to share care with others. Local authorities are the lead agencies for assessing need and arranging care. Increasingly, other families are recruited to offer respite or longterm care, but it must be recognised that these families may need similar support to that offered to the family of origin.

\section{Meeting personal and family needs}

People with learning disabilities need a home and family life, education, vocational training and employment, leisure and social activity, and health care. Most of these are the same needs as everyone else, but people with learning disabilities may need additional support and individually-designed programmes of personal and social development. Families may also need information, advice, financial support and respite care. The level of support required from services depends on the person's ability, their family's coping skills, and the other sources of support available to them. Individuals who need specialised services because of complex disabilities need primary care services too.

The types of service needed do not show a clear division between education, social care and health care; nor do they show a division between primary care and secondary care. At best, this could mean that a person's needs are met holistically, in a seamless service. Some needs are mainly social, such as employment. Examples of needs which are mainly related to health are shown in Box 4.

\section{Service models}

The pattern of services offered to people with learning disabilities embodies the philosophy of care which is current at the time each service is established. Elements of previous models of care persist and are adapted to newer models of care.
Box 4. Examples of health needs of learning disabled individuals

Advice on diet, eating and weight problems

Advice on dental hygiene and dental problems

Advice on menstrual hygiene and menstrual problems

Advice about 'lifestyle' risks - smoking, alcohol abuse, less safe sexual activity

Ensuring supported access to health programmes in primary care-immunisation, screening for breast and cervical cancer

Detecting and responding to additional needs (e.g. communication problems, behavioural problems, mobility and sensory problems)

Screening for problems associated with Down's syndrome (e.g. hypothyroidism, unstable cervical spine)

Detecting, diagnosing and treating illness and being aware of common conditions such as epilepsy or mental illness

Contraceptive advice, prescription and monitoring

Rational prescribing, e.g. monitoring levels of anti-epileptic and other medication

Changes have also occurred in primary care, mainly in response to changes in specialist provision.

\section{Use of primary care}

Despite some contrary findings, most studies find that GP consultation rates are low (Wilson \& Haire, 1990). Some GPs may assume that the general medical care of patients with learning disability is the responsibility of someone else (Barker \& Howells, 1990), or at least that their needs are being catered for elsewhere (Ineichen \& Russell, 1980).

The number of patients with learning disability on the list of an average GP has been estimated as $30-40$ with mild disability and 6-8 with severe disability, across the age range, including 2-3 people still living in hospital (Livingstone, 1990). There has been an entitlement to GP services by people in the community since the inception of the NHS, and some people have assumed that the numbers are so small that the closure of mental handicap hospitals will make no difference. Resettlement plans have rarely included primary care, yet studies of the effects of resettlement show that GPs are the professionals in contact with the highest proportion of resettled people (De Paiva \& 
Lowe, 1990). Evans et al (1994) showed that, between 1986 and 1992, rates of contact with GPs rose from $59 \%$ to $69 \%$, while rates of contact with all others except dentists were much lower. Contact rates with community nurses moved from $17 \%$ to $20 \%$, and contact with psychiatrists, social workers and professions allied to medicine remained static or fell. Whether planned or not, people returning to the community use their GP as the commonest source of professional advice.

\section{Communication between primary and secondary care}

The referral letter, as the traditional method of communication between GP and specialist, may have been appropriate to a service model in which secondary care services removed people entirely from primary care, but it is unlikely to deliver the best health care in present services. Box 5 shows possible ways of helping GPs to improve the service for their patients.

\section{Closing the gap between primary and secondary care}

The World Health Organization (1994) seeks to create a better partnership between primary health care and social care on the one hand, and specialist mental health care on the other, so as "to optimise the assessment, treatment and rehabilitation of people with mental disabilities in order to achieve comprehensive care".

The history of separation of primary and secondary care, with segregated specialist services, has led to the situation where there is rarely any organisational relationship for planning services at the interface between primary and secondary care, and it is hard to identify a strategy to meet health care needs (Langan et al, 1993).

The majority of people with learning disabilities have always been supported in integrated community settings by their families. Meeting the needs of people with severe complex disabilities in integrated community services can work better than other service models if care staff get to know an individual very well - both in terms of the documented history and also their subjective experience; and if there is a continuous high commitment by senior management, with an investment in diversity of provision. High quality management of direct-care staff is crucial, and there must be ready access to specialist services.
The Mansell report advised that the capability of 'generic' services should be developed to meet the needs of people with learning disabilities (Department of Health, 1993), interpreted by some to mean that specialised services are no longer required. The Department of Health has confirmed that specialised services are still needed. Services which are successful sometimes appear to be doing nothing extraordinary. They use ordinary-looking facilities, and behave towards people in apparently ordinary ways. It takes a high level of skill and organisation, and adequate resources, to achieve success. Specialised services which appear 'ordinary' may be vulnerable to changes of staff, changes in organisations, and 'asset stripping'. Seen in this light, segregation may only be a substitute for adequate specialisation.

\section{Improving the health of people with learning disabilities}

Health policy in the UK applies equally to people with learning disabilities. For example, the 'Health of the Nation' policy includes targets to reduce illhealth and death caused by mental illness (Department of Health, 1995). People may need support to use services, to ensure equity of services, and to achieve equal outcomes. Three ways of improving

Box 5. Developing the competence of primary care teams

Undergraduate training:

- booklets and 'handouts'

- videos

- family visits

Postgraduate training:

- listening to local GPs and primary care teams about how they see their training needs

- distance learning materials

- workshops

Advice about individuals:

- joint consultation

- regular meetings between GPs and specialists

- ad hoc meetings or phone calls to discuss patient care

- case presentation

Advice about programmes:

- checklists for detecting disability

- protocols for health surveillance 
health are sometimes described as health promotion, health surveillance and health care.

\section{Health promotion}

One aim of health promotion is to reduce the occurrence of avoidable learning disability - for example, by encouraging immunisation, sensible drinking during pregnancy, and age-related family planning. Helping people with learning disabilities to choose a healthy way to live cannot be done entirely by the health service, but doctors can use their knowledge to influence other services. Some topics are relevant to primary care - for example, ensuring that families of people with Down's syndrome are aware of the possibility of Atlantoaxial instability and the risk posed by sudden neck flexion. Other topics are mainly relevant to specialist learning disability services - for example, policies to prevent financial, emotional, physical, or sexual abuse. At the interface, specialist staff such as community nurses can help their clients to understand and use primary care services. The Royal College of Nursing (1994) has suggested that Community Learning Disability Nurses could become part of the primary care team.

\section{Health surveillance}

Primary care is organised mainly as an on-demand service, but people with learning disabilities are low users of primary care, despite high rates of illness. Early detection of treatable illness improves the quality of life for the carers as well as the patient. To achieve full coverage, health surveillance requires a register, which is usually organised by specialist services, although some exist in primary care. For many kinds of illness, the best approach is to arrange support for the individual to use programmes for the rest of the population such as breast screening. Protocols have been devised as a basis for health surveillance, for example see Howells \& Barker (1990).

\section{Health care}

Box 6 lists some of the reasons why it is difficult to ensure that people with learning disabilities receive the same standard of health care as everyone else.

Specialist health care for most medical and surgical problems is provided through the secondary care services available to the rest of the population. Psychiatry of learning disability aims to provide specialist health services for people with learning disabilities who have mental illness, epilepsy, or behavioural problems, or who may have contact with the criminal justice system.

Other specialist learning disability services are also provided by the NHS. Services described as 'habilitative' are often provided by learning disability services, including physiotherapists, clinical psychologists, speech therapists, occupational therapists and nurses. Clients of the service are not necessarily under the care of a consultant, and this may not be clear to the GP. Specialist community health services are usually provided through secondary care organisations, which may have developed from mental handicap hospitals. Those hospitals often took responsibility for the general health care of their residents, and it is not surprising that some GPs are confused about who should be responsible for the continuing health care of former residents. General practitioners have primary medical responsibility for anyone who is registered with them, once they have been informed that the person has left hospital (Department of Health, 1989).

Box 6. Barriers to good health care for people with learning disabilities

They may not recognise symptoms as an indicator of illness

They may not know about the possibility of help through a doctor

They may not report their symptoms

Carers may not realise the significance of symptoms or think symptoms are severe enough to warrant medical attention, and may not provide the support needed for the person to consult a doctor

They may be unable to describe symptoms clearly to a doctor or to give answers that a doctor needs

They may not understand about the need for examination, and may not cooperate

It may be unclear whether the problem is due to illness or some other cause

They may be offered different treatment because of difficulty in obtaining consent, or because of predictions about how they would cooperate or react to treatment

Treatment intended for short-term use may not be reviewed, because carers request repeat prescriptions

GPs may not be aware of ways in which learning disability services could help them provide a better service 
Primary care staff may feel de-skilled, and believe that they have nothing to offer except referral to secondary care. Secondary care teams might produce information packs or checklists to help primary care teams to develop appropriate skills and to be more effective.

Access may be facilitated in various ways. All health services could ensure that physical access is good (for example, with ramps) and that acoustics and lighting are good. The system for appointments and waiting rooms could be made as simple and explicit as possible.

Staff from community learning disability teams could help to prepare patients for consultation, for example by video or role play about the process of examination. They could support the person during the interview, acting as an interpreter to help the person understand the doctor and vice versa, or else they could help the person to find out the information the doctor needs, such as keeping a diary or chart of symptoms. They can also act as informants, if necessary.

\section{Audit and research}

It is difficult to arrange audit to bridge primary and secondary care. Creating a peer group to agree shared and measurable standards is a formidable undertaking. There are some aspects of medical care that are commonly shared, in particular, the prescription of long-term antipsychotic or antiepileptic medication. People with learning disabilities are vulnerable in several ways; they have high rates of the disorders requiring these drugs, they are vulnerable to adverse effects such as tardive dyskinesia, they may not control their own medication, and they may have less ability to report adverse effects. Audit of these topics could improve medical care substantially.

The Department of Health (1994) has identified the interface between primary and secondary care as an important area. Rehabilitation and community care for priority groups, including learning disability, is a priority for the NHS R\&D programme.

\section{References}

American Psychiatric Association (1994) Diagnostic and Statistical Manual of Mental Disorders (4th edn) (DSM-IV). Washington, DC: APA.

Barker, M. \& Howells, G. (1990) The medical needs of adults. In Primary Care for People with a Mental Handicap. Occasional Paper 47, pp. 6-8. London: Royal College of General Practitioners.

British Paediatric Association (1994) Services for Children and
Adolescents with Learning Disability (Mental handicap). Report of a Working Party. London: British Paediatric Association.

De Paiva, S. \& Lowe, K. (1990) The Evaluation of NIMROD, a Community-based Service for People with Mental Handicap; Client's Uses of Services. Mental Handicap in Wales Applied Research Unit Report (quoted in Langan et al, 1993).

Department of Health (1989) Medical Responsibility in NHS Hospital and Community Services for Mentally Ill and Mentally Handicapped People PL/CMO(89)1. London: Department of Health.

- (1991) Statement on Services of People with Learning Disabilities. Stephen Dorrell's Speech to Mencap, June 1991. London: Department of Health.

- (1993) Services for People with Learning Disabilities and Challenging Behaviour or Mental Health Needs. Report of a Project Group (Chairman: Prof. J. L. Mansell). London: Department of Health.

- (1994) RED Priorities in Relation to the Interface Between Primary and Secondary Care. London: Department of Health.

- (1995) The Health of the Nation: a Strategy for People with Learning Disabilities. London: HMSO.

Evans, G., Todd, S., Beyer, D., et al (1994) Assessing the impact of the All-Wales Mental Handicap Strategy: a survey of four districts. Journal of Intellectual Disability Research, 38, 109-133.

Fryers, T. (1991) Recent epidemiological studies in mental retardation. Current Opinion in Psychiatry, 4, 662-666.

Hall, D. M. B. (1984) The Child with a Handicap. Oxford: Blackwell.

Howells, G. \& Barker, M. (1990) A protocol for primary health care. In Primary Care for People with a Mental Handicap. Occasional Paper 47, 12-13. London: Royal College of General Practitioners.

Ineichen, B. \& Russell, O. (1980) Mental Handicap and Community Care - the Viewpoint of the General Practitioner. Mental Handicap Studies - Research Report No. 4. Bristol: University of Bristol.

Langan, J., Russell, O. \& Whitfield, M. (1993) Community Care and the General Practitioner: Primary Care for People with Learning Disabilities. Research Report to the Department of Health. London: Department of Health.

Livingstone, D. (1990) Mentally handicapped people, community care and the general practitioner. In Primary Care for People with a Mental Handicap. Occasional Paper 47, pp. 2-3. London: Royal College of General Practitioners.

Royal College of Nursing (1994) Learning Disability Nursing: an RCN Guide for Purchasers. London: Royal College of Nursing.

Wilson, D. \& Haire, A. (1990) Health care screening for people with mental handicap living in the community. British Medical Journal, 301, 1379-1381.

World Health Organization (1992) The ICD-10 Classification of Mental and Behavioural Disorders: Clinical Description and Diagnostic Guidelines (ICD-10). Geneva: WHO.

- (1994) Statement by EURO Meeting of National Directors/ Officials of Mental Health Services in the European Member States of WHO. Kellokoski, Finland, 1-3 December 1994. Geneva: WHO.

\section{Multiple choice questions}

1 Mental retardation

a was used in ICD-9 but has been dropped from ICD-10

b includes autism

c does not have a defined maximum age of onset

$\mathrm{d}$ is the preferred term in the UK

e is a useful concept for epidemiological use

2 The detection of learning disability

a sometimes leads to diagnosing a remediable cause 
b usually takes place in the neonatal period

c should be delayed as long as possible

$\mathrm{d}$ may be improved by using published checklists

e means that responsibility for health care passes to specialists in learning disability

3 These statements about epidemiology are true a about $2 \%$ of the population have a learning disability

b A GP's list of 2000 will have one child with severe learning disability born in the practice yearly

c about $50 \%$ of people with learning disabilities have mild learning disability.

d autism is associated with epilepsy

e at one time, most people with learning disabilities lived in institutions
4 Primary care teams

a may employ Community Learning Disability Nurses

b have little to offer families except referral to specialists

c can be more effective if Community Teams prepare the patient for an examination

d could conduct audit of long-term prescribing jointly with specialists

e sometimes use checklists or protocols suggested by specialists

\begin{tabular}{|c|c|c|c|}
\hline \multicolumn{4}{|c|}{ MCQ answers } \\
\hline 1 & 2 & 3 & 4 \\
\hline a $F$ & a $\mathrm{T}$ & a $\mathrm{T}$ & a $\mathrm{T}$ \\
\hline b F & b F & b F & b F \\
\hline c $\mathrm{T}$ & c $F$ & c F & c $\mathrm{T}$ \\
\hline d F & d $T$ & d $\mathrm{T}$ & d $T$ \\
\hline e $T$ & e $F$ & e $F$ & e $T$ \\
\hline
\end{tabular}

\section{Correspondence}

\section{Preparing a medico-legal report}

SIR: The excellent article by Bluglass (APT, May 1995, 1, 131-137) highlights a challenge for the Editor if Advances in Psychiatric Treatment is to become the major journal of continuing professional development for psychiatrists throughout the British Isles. Bluglass acknowledges the "differences between the jurisdictions of the United Kingdom" but then deals exclusively with the English Mental Health Act and the English legal system. Practising in a country with no coroner's courts, Mental Health Act Commission or mental health review tribunals, and a Mental Health Act that does not extend to psychopathic disorder, most of the specific issues requiring a medico-legal report discussed by Bluglass are, at best of no relevance to me, and at worst confusing and misleading.

How should the different legal and administrative systems in Scotland, Northern Ireland and the Republic of Ireland be covered? The editors of Seminars in Practical Forensic Psychiatry (Chiswick \& Cope, 1995) have probably struck the right balance. Relevant chapters have appendices written by psychiatrists practising in the rest of the British
Isles setting out the legal and administrative arrangements pertaining to their country or province. The chapter is therefore of educational value to those practising outside England. Could a similar system be adopted by APT?

Chiswick, D. \& Cope, R. (eds) (1995) Seminars in Practical Forensic Psychiatry. London: Gaskell.

I. Pullen

Borders Community Health Services NHS Trust

Dingleton Hospital

Melrose

Roxburghshire TD6 9HN

EdTOR's RePLY: Dr Pullen makes an important point and in future Advances in Psychiatric Treatment will aim to have relevant information for other parts of the British Isles when there is specific reference to English law. I wonder if readers in England and Wales would have other requests for articles in $A P T$ more geared to their requirements, for example, on management aspects of psychiatry? We welcome correspondence and we also welcome suggestions for future articles.

A. C. P. SiMS 